\title{
Ampliación de ámbito de la serpiente Agkistrodon bilineatus (Serpentes: Viperidae), en el Sureste de Honduras
}

\author{
Julio Enrique Mérida ${ }^{1}$, Ramón Dionicio Centeno ${ }^{1,2}$ y Gustavo Adolfo Cruz ${ }^{1}$ \\ ${ }^{1}$ Museo de Historia Natural, Universidad Nacional Autónoma de Honduras, Bulevar Suyapa, Tegucigalpa, Francisco Morazán, Honduras; \\ juliomerid@yahoo.com \\ ${ }^{2}$ Fundación Yuscarán, Yuscarán, El Paraíso, Honduras; fundacionyuscaran2005@yahoo.es
}

Recibido 15-II-2012 Corregido 8-III-2012 Aceptado 17-IV-2012

\begin{abstract}
Agkistrodon bilineatus was known to occur in Honduras in four localities in the departments of Choluteca, Valle and Copan. Here we add Orealí (El Paraiso), near the "Yuscarán Biological Reserve." Previous surveys suggested that this species was absent from the Honduras Pacific. These specimens are assigned to the subspecies Agkistrodon bilineatus howardgloydi based on scales and tail length.
\end{abstract}

\section{KEY WORDS}

Water Moccasin, cliff, Agkistrodon bilineatus howardgloydi, Honduras, Yuscarán Biological Reserve.

\section{RESUMEN}

De Agkistrodon bilineatus se mencionan registros en Honduras en cuatro localidades correspondientes a los departamentos de Choluteca, Valle y Copán. Aquí se agrega la localidad de Orealí, en el departamento de EI Paraíso, cerca del Área Protegida "Reserva Biológica Yuscarán". Muestreos previos sugerían que esta especie ya no tenía presencia en el Pacífico de Honduras. Estos especímenes se asignan a la subespecie Agkistrodon bilineatus howardgloydi por escamación y longitud de cola.

\section{PALABRAS CLAVE}

Mocasín de agua, cantil, Agkistrodon bilineatus howardgloydi, Honduras, Reserva Biológica de Yuscarán.
El género Agkistrodon se distribuye desde el noreste, centro y sureste de Estados Unidos, hacia el suroeste de Texas y norte de Coahuila, México, y hasta Centro América (McCraine 2011). Este género contiene 4 especies formalmente descritas (Knigth 1991, Savage 2002). De las cuales $A$. bilineatus se encuentra en elevaciones bajas a moderadas a lo largo de la Costa del Pacífico, desde el sur de Sonora en México hacia el sur a través de Guatemala, El Salvador y Nicaragua, hasta el noroeste de Costa Rica y el Norte de Yucatán hasta los 1500 msnm (Köhler 2003).

A. bilineatus, comprende las siguientes subespecies: A. $b$. russeolus distribuida en Yucatán y Belice, A. $b$. bilineatus desde la vertiente del Pacífico de México y Centro América, del Sureste de Sonora hasta el Sur de El Salvador, A. b. howardgloydi que ocurre en Honduras, Nicaragua y Costa Rica, y A. b. lemosespinali descrita por Smith y Chizar (2001) de Palma Sola, Veracruz México. En cuanto a la subespecie A. b. taylori Parkinson et al. (2000) proponen cambio a la categoría de especie, y por lo que se consideran aquí solo cuatro subespecies.

El primer registro en Honduras de Agkistrodon fue publicado por Cruz et al. (1979) en la isla Zacate Grande, Wilson y Meyer (1982). Solórzano et al. (1999), mencionan que los esfuerzos realizados por Mahmood Sasa (su coautor) no proporcionaron especímenes, en Honduras. McCraine (2011) también menciona que no pudo obtener reconocimientos positivos mediante entrevistas con fotografías mostradas a colectores profesionales de reptiles en El Madreal, Departamento de Choluteca. Este autor presenta cuatro localidades en Honduras, de las que dos representan material que el no revisó. (Fig. 1).

Hay registros de esta especie en el Departamento de Choluteca y Valle, en el extremo sur del país, y en el borde con Guatemala, Departamento de Copán. En la presente 


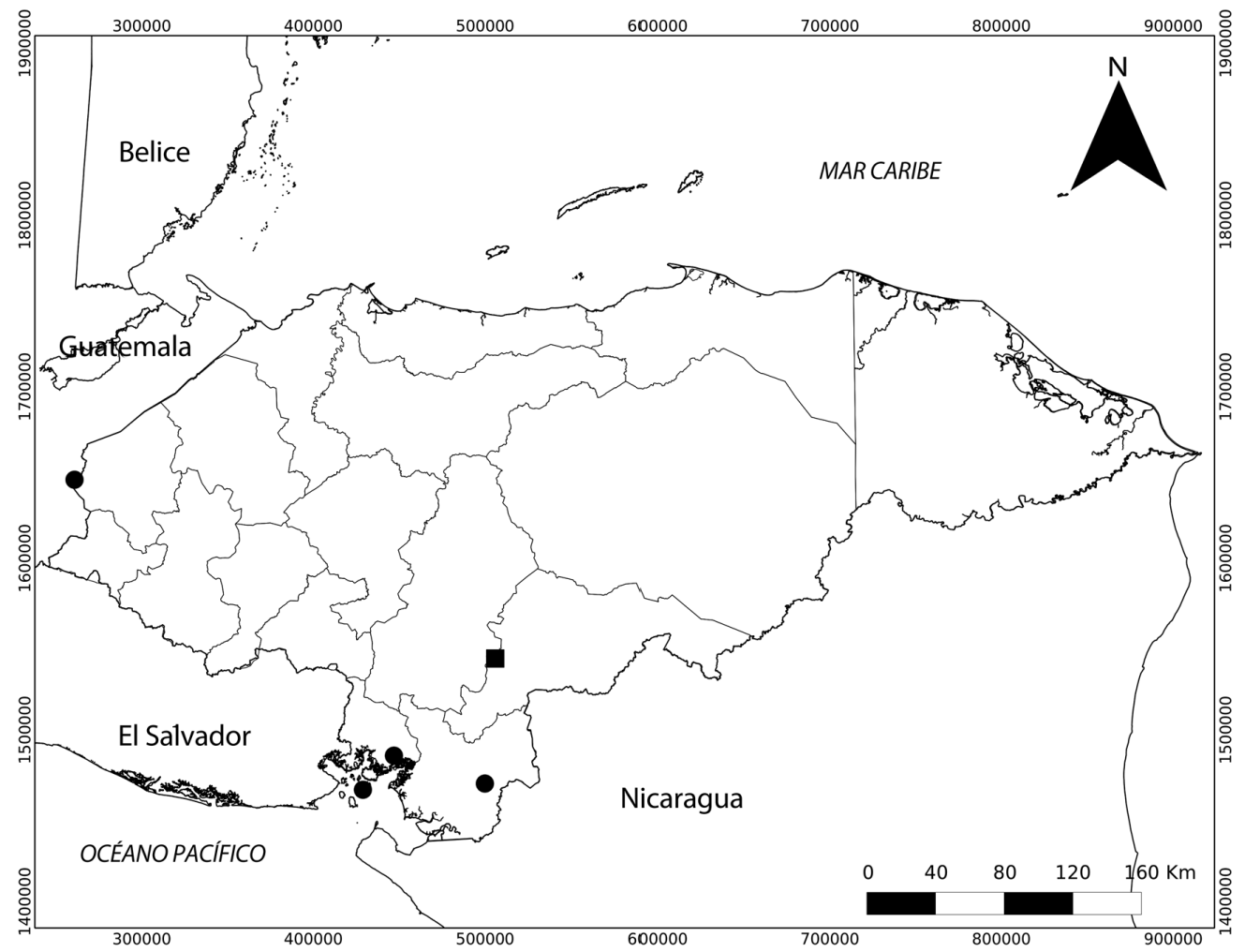

FIG. 1. Registro de localidades de Agkistrodon bilineatus en los Departamentos de Honduras, los círculos representan previas localidades mostradas en McCraine (2011) para los Departamentos de Copán, Valle y Choluteca, el cuadrado indica el nuevo reporte de localidad, Orealí Departamento de El Paraíso.

nota se incluye una nueva localidad de distribución en el Departamento del Paraíso entre los 400 y 450 msnm.

\section{Descripción de especímenes}

UNAH 5649, 20 de Septiembre 2011; hembra LT $340 \mathrm{~mm}$, juvenil. UNAH 5650, 8 de Noviembre 2011, hembra, $840 \mathrm{~mm}$, adulto, colectados en la comunidad de Orealí por R. D. Centeno. Ambos presentan 9 placas de escamas dorsales en la cabeza; nasal dividida; una loreal; prelacunal presente; 2 suboculares; 2 preoculares en juvenil y 3 en adulto; 2 postoculares; 8 supralabiales; 11 infralabiales, las primeras dos en contacto en la parte posterior medial de la mental, canal mental presente; 133 ventrales en juvenil y 134 en adulto; cloacal entera; subcaudales en juvenil 65 (23 completas y 42 divididas), en adulto 65 (18 completas y 47 divididas); escamas dorsales quilladas, en filas del cuerpo 27-23.20 en juvenil, 26-23-18 en adulto. Cola 20 por ciento de la longitud total en juvenil (71.37), y 19 por ciento en adulto $(160 \mathrm{~mm})$.
El patrón de coloración y los conteos de escamas de acuerdo a los datos presentados por Cruz et al (1979), Conant (1984), Campbell y Lamar (1989) y Savage (2002), así como el ámbito de distribución presentado por Conant (1984) indican que los especímenes reportados aquí corresponden a la subespecie $A$. b. howardgloydi.

\section{Localidades}

El primer registro corresponde a 13052'4.4" Latitud y $86^{\circ} 47^{\prime} 1.5^{\prime \prime}$ Longitud a $418 \mathrm{msnm}$, y el segundo registro corresponde a 13०52'13.4" Latitud y 86²'32.2" Longitud a $452 \mathrm{msnm}$. ambos en la aldea de Orealí, El Paraíso, en los límites de la Reserva Biológica de Yuscarán; esta localidad están dentro del ámbito de distribución propuesto para A. b. howardgloydi.

\section{Comentarios}

El primer espécimen fue eutanizado y colectado tras morder en la mano a un adulto mayor, el cual fue tratado 


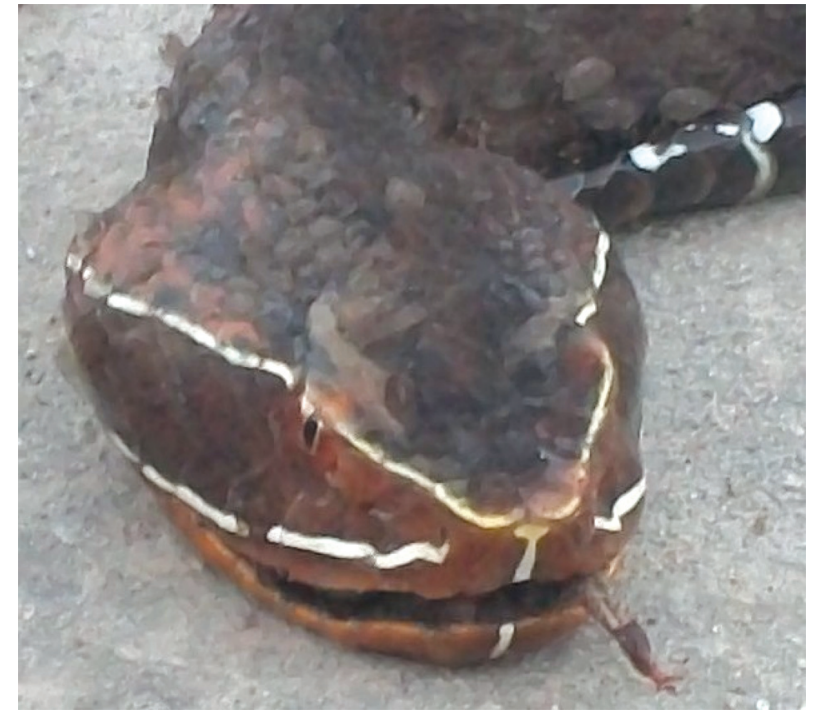

FIG. 2. Cabeza de Agkistrodon bilineatus howardgloyidi, hembra adulta, mostrando las dos líneas.

Fotografía de R.D. Centeno.

con suero antiofídico tres horas después, logrando sobrevivir. A. bilineatus se encuentra amenazado a través de su ámbito, debido a la transformación del bosque deciduo tropical y matorral por la agricultura y otras actividades antropogénicas. A. bilineatus se considera como Casi Amenazado en la Lista Roja de la IUCN (Lee \& Hammerso, 2007) y en la categoría II de CITES. Es necesario estudiar el estado de las poblaciones de esta especie en Honduras, y debe tener una atención especial por parte de la Fundación Yuscarán que administra la Reserva Biológica Monserrat, y por el Estado de Honduras a través del Instituto Nacional de Conservación y Desarrollo Forestal, Áreas Protegidas y Vida Silvestre (ICF). Recomendamos expandir el límite de la reserva biológica con el objetivo de proteger y conocer sobre la historia natural de esta especie en Honduras.

\section{AGRADECIMIENTOS}

Agradecimiento especial a la Fundación Yuscarán por el apoyo logístico y al "Proyecto Ecosistemas" por su apoyo económico para el desarrollo de la práctica profesional de Ramón D. Centeno; A Saúl Flores Asistente Curador del Museo de Historia Natural, al personal voluntario Wilbert del Cid, Enmanuel Meraz, Josué Ramos, Thelma Salgado por su ayuda y asistencia. Klaus Wiese por la elaboración del mapa, a José Cristobal López Brooks, Tania Najarro y Zenia Salinas por su ayuda para obtener literatura especializada.

\section{REFERENCIAS}

Campbell, J.A. \&W.W. Lamar. 1989. The venomous Reptiles of Latin America. Cornell University, Ithaca, Nueva York, EEUU.

Conant, R. 1984. A new subspecies of the Pit Viper, Agkistrodong bilineatus (Reptilia: Viperidae) From Central America. Proceedings of the Biological Society Washington. 97:135-141.

Cruz Díaz, G.A., L.D. Wilson \& J. Espinosa. 1979. Two additions to the reptiles fauna of Honduras. Eumeces managuae Dunn and Agkistrodon bilineatus (Günther). With comments on Pelamis platurus (Linnaeus) Herpetological Review. 10:26-27.

Köhler, G. 2003. Reptiles de Centro América. Offenbach, Alemania.

Knight, R.A., Jr. 1991. Molecular Systematics of the Agkistrodon Complex. Thessis Philosophy degree. Texas Tech University in Partial Fulfillment of the Requirements for the Degree of Doctor of Philosophy, Texas, EEUU.

Lee, J. \& G.A. Hammerson. 2007. Agkistrodon bilineatus. IUCN 2011. IUCN Red List of Threatened Species. Version 2011.1.

McCranie, J.R. 2011. The Snakes of Honduras. Systematics, Distribution, and Conservation. Contributions to Herpetology, Volume 26. Society for the Study of Amphibians and Reptiles. Thomson-Shore, Michigan, EEUU.

Parkinson, C.L., K.R., Zamudio \& H.W. Greene. 2000. Phylogeography of the pitviper clade Agkistrodon: historical ecology, species status, and conservation of cantils. Molecular Ecology 9: 411-420.

Savage, J.M. 2002. The Amphibian and Reptiles of Costa Rica: A Herpetofauna between two continents, between two seas. University of Chicago, Chicago, EEUU.

Smith, H.M. \& D. Chiszar. 2001. A new subspecies of cantil (Agkistrodon bilineatus) from central Veracruz, México. Bulletin of the Maryland Herpetological Society 37: 130-136.

Solorzano, A., Romero, M., J. María Gutiérrez \& M. Sasa. 1999 Venom Composition and Diet of the Cantil Agkistrodon bilineatus howardgloydi (Serpentes: Viperidae). The Southwestern naturalist 44 : 478-483.

Wilson. L.D. \& J.R. Meyer. 1982. The Snakes of Honduras. Milwaukee Public Museum, Milwaukee, Wisconsin, EEUU. 